\title{
Mucormicosis rino-órbito-cerebral con manifestación palatina en un paciente diabético. A propósito de un caso clínico y revisión de la literatura
}

\author{
Rhino-orbit-cerebral mucormycosis with palatal manifestation, in a diabetic \\ patient. Apropos of a clinical case and review of the literature
}

\author{
Alejandro Alonso Moctezuma,*, Edmundo Santos Jaimes, ${ }^{*, \S}$ \\ Eric Zeta Castañeda, *, Er Erick Reyes Castañeda, IJavier Portilla Robertson*,***
}

\section{RESUMEN}

La mucormicosis es una infección rara, aguda y frecuentemente mortal, es causada por hongos aerobios saprófitos y oportunistas de la subdivisión Mucoromycotina (antes Zygomycetes). Se han descrito varias formas clínicas: rino-órbito-cerebral, pulmonar, gastrointestinal, mucocutánea y diseminada, la mucormicosis rino-órbitocerebral (MROC) con frecuencia se asocia con pacientes diabéticos descompensados o con enfermedades hematológicas. La forma rino-órbito-cerebral es de rápida progresión y alta morbimortalidad en los pacientes inmunosuprimidos con necesidad de manejo médico y quirúrgico multidisciplinario. Se reporta el caso de un paciente con mucormicosis rino-órbito-cerebral asociada con diabetes

\section{ABSTRACT}

Mucormycosis is a rare, acute and frequently lethal disease caused by aerobic saprofit fungi and opportunists of the Mucoromycotina subdivision (before Zygomicota). Several clinical presentations have been described: rhino-orbitalcerebral, pulmonar, gastrointestinal, mucocutaneous and disseminate, rhino-orbital-cerebral mucormycosis (ROCM) is frequently associated To diabetic patients with inadequate control or hematological diseases. The rhinoorbital-cerebral presentation has a quick development with high morbidity and mortality in immunocompromised patients, with the need of a multidisciplinary medical and surgical approach. Case report of a patient with rhinoorbital-cerebral mucormycosis associated to uncontrolled

\footnotetext{
* División de Estudios de Postgrado e Investigación de la Universidad Nacional Autónoma de México. Ciudad de México, México.

₹ Coordinador de la Especialidad en Cirugía Oral y Maxilofacial.

$\S$ Adscrito al Departamento de Patología Bucal y Maxilofacial.

" Residente del Departamento de Patología Bucal y Maxilofacial.

" Adscrito. Cirugía Maxilofacial. Centro Médico «Lic. Adolfo López Mateos». Toluca de Lerdo, Estado de México.

** Coordinador del Departamento de Patología Bucal y Maxilofacial. 
mellitus tipo 2 descompensada, con afección palatina y cutánea, tratada quirúrgicamente con maxilectomía total bilateral, con seguimiento de 16 meses sin evidencia de recidiva.

Palabras clave: Mucormicosis rino-órbito-cerebral, diabetes mellitus, inmunosupresión. diabetes mellitus DM2, palatine and cutaneous invasion, who was surgically treated with total bilateral maxillectomy, showed no evidence of recurrency in the next 16 months of follow up.

Keywords: Rhino-orbital-cerebral mucormycosis, diabetes mellitus, immunosuppression.

\section{INTRODUCCIÓN}

Esta enfermedad fue descrita en el siglo XIX por Paltauf, a la que llamó «micosis mucorina»; sin embargo, el agente causal no fue aislado, por la morfología de los filamentos observados en los tejidos dichos cuadros infecciosos fueron llamados mucormicosis.

Se presenta en todo el mundo, es una infección micótica con gran potencial de invasión aguda, frecuentemente mortal causada por hongos oportunistas. Entre los agentes responsables de la mucormicosis se encuentran principalmente los del subtipo de Mucoromycotina en el orden mucorales..$^{1-3}$

Esta infección está asociada con alteraciones sistémicas, en países en vías de desarrollo se ha observado su relación con diabetes mellitus no controlada, mientras que en países desarrollados se relaciona con neoplasias hematológicas como leucemia y otras condiciones como trasplante de células madre hematopoyéticas o trasplante de órganos sólidos. Sin embargo, existen reportes de mucormicosis en pacientes inmunocompetentes, pero con presencia de áreas de traumas o quemaduras en la piel. ${ }^{2-4}$

El agente causal de la mucormicosis es un organismo saprofito, generalmente crece en el suelo en materia orgánica en descomposición como frutas, verduras, pan y semillas. Los mucorales son aerobios, pero pueden sobrevivir en condiciones microaerofílicas, y como cualquier tipo de hongo se nutren por absorción. La mayoría de los mucorales se desarrollan bien a temperaturas de 30 a $40^{\circ} \mathrm{C}$. Sus esporas son liberadas en el aire, pueden ser inhaladas o inocularse directamente en la piel con alguna alteración. El género más frecuente en la mucormicosis es el género Rhizopus; sin embargo, podemos encontrar algún otro hongo agregado..$^{2,5}$

La fisiopatogenia en los pacientes diabéticos descompensados se fundamenta en las alteraciones propias de la enfermedad de base como las mucosas secas, condición que facilita la adherencia y permanencia de las esporas sobre el tejido. El metabolismo de cuerpos cetónicos por Rhizopus arrhizus es lo que favorece su desarrollo in situ. La hipertermia frecuente y la gran cantidad de glucosa en tejidos y exudados proporciona condiciones propicias para el rápido crecimiento de las estructuras filamentosas que se unen a los vasos sanguíneos, los penetran y obstruyen causando trombosis en cuestión de días, lo que provoca áreas de necrosis. Rhizopus $s p$. son capaces de adherirse a las células endoteliales y causar lesión aun cuando el hongo ya haya muerto. ${ }^{5,6}$

Se han descrito diferentes formas clínicas, las más frecuentes son la rino-órbito-cerebral aguda y la mucormicosis pulmonar, con menos frecuencia la forma cutánea primaria y gastrointestinal. Esta enfermedad presenta altas tasas de morbimortalidad y su manejo requiere un equipo multidisciplinario. ${ }^{5,6}$

El propósito de este artículo es describir la fisiopatología, diagnóstico y tratamiento médico-quirúrgico de un caso clínico de mucormicosis rino-órbitocerebral.

\section{CASO CLÍNICO}

Paciente masculino de 58 años acude a consulta al Departamento de Patología Bucal y Maxilofacial en la División de Estudios de Postgrado e Investigación de la UNAM por presentar una úlcera en paladar con dos semanas de evolución. Refirió ser diabético mal controlado y haber padecido un cuadro de neumonía y parálisis facial cuatro meses antes de su consulta inicial en nuestro servicio; fue tratado por dos médicos privados con antisépticos bucales y múltiples antibióticos sin presentar mejoría. A la exploración física presentó una úlcera en paladar duro del lado izquierdo de $1.5 \mathrm{~cm}$ de diámetro con fondo de aspecto necrótico color blanco-amarillento con áreas color café y exposición ósea en el centro, bordes irregulares con áreas eritematosas y zonas blancas consistentes con fibrina, asintomática (Figura 1). Se solicita tomografía computarizada en la que se observan zonas hiperdensas rodeadas por halos hipodensos en la apófisis palatina del hueso maxilar, 


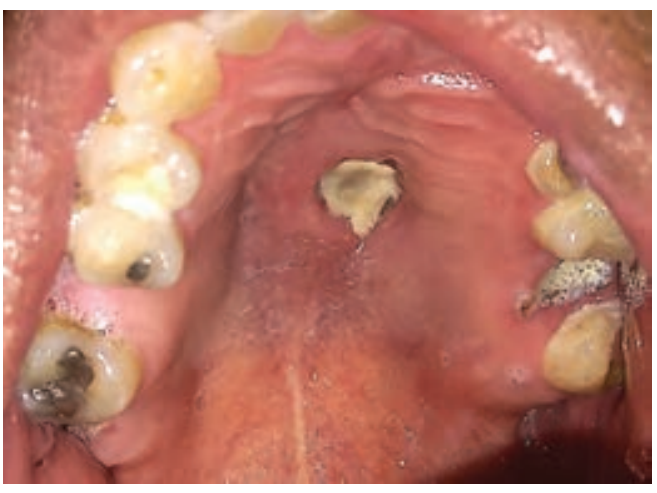

Figura 1: Úlcera localizada en paladar duro del lado izquierdo de $1.5 \mathrm{~cm}$ de diámetro. Se observa fondo de aspecto necrótico color blanco-amarillento con áreas color café, en el centro se aprecia exposición ósea con bordes irregulares y eritematosos.

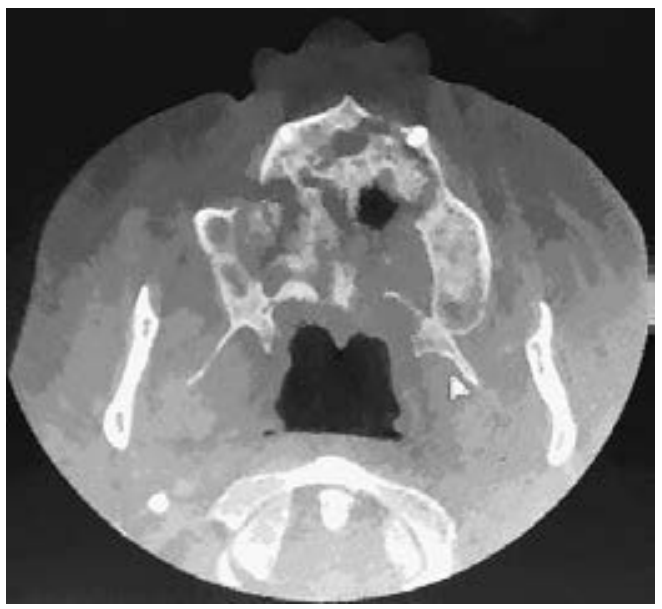

Figura 2: Tomografía axial en un corte transversal en la que se observan zonas hiperdensas rodeadas por halos hipodensos en la apófisis palatina del hueso maxilar. también se observó perforación de la pared anterior del seno maxilar y engrosamiento de la membrana sinusal (Figura 2). Se decide tomar biopsia incisional de los bordes de la úlcera y del hueso expuesto, previa valoración y control metabólico por parte de médico internista.

En los cortes histológicos examinados se observa proliferación de estructuras celulares alargadas, cilíndricas, no septadas, algunas con formaciones en $Z$ con ángulos de 90 grados, compatibles con hifas, tienen características de degeneración, además una gran cantidad está en proceso de gemación en un tejido conjuntivo fibroso entremezclado con tejido óseo no vital. Se realizaron tinciones de histoquímica con PAS y Grocott positivos. Con base en estos hallazgos se emite diagnóstico de mucormicosis (Figura 3).

Con los hallazgos clínicos, imagenológicos e histopatológicos se emitió el diagnóstico de mucormicosis rino-órbito-cerebral, por lo que se decide iniciar tratamiento con anfotericina $\mathrm{B}$ y programar para maxilectomía en el Centro Médico «Lic. Adolfo López Mateos» del ISEM; sin embargo, el paciente toma la decisión de no ingresarse y abandona el tratamiento propuesto para buscar terapias alternativas. Se presenta a consulta una semana después con secreción purulenta a través de la úlcera, conducto auditivo izquierdo y con presencia de múltiples abscesos cutáneos en hemicara izquierda. El paciente acepta el tratamiento propuesto anteriormente y se ingresa al Servicio de Cirugía Maxilofacial. Se solicita interconsulta del Servicio de Medicina Interna para control de glicemia y de infectología para tratamiento farmacológico. Se inició tratamiento con anfotericina b deoxicolato a dosis diarias calculadas a $1 \mathrm{mg} / \mathrm{kg} /$ día, al día siete se programó y realizó maxilectomía total bilateral según la clasificación de Cordeiro,

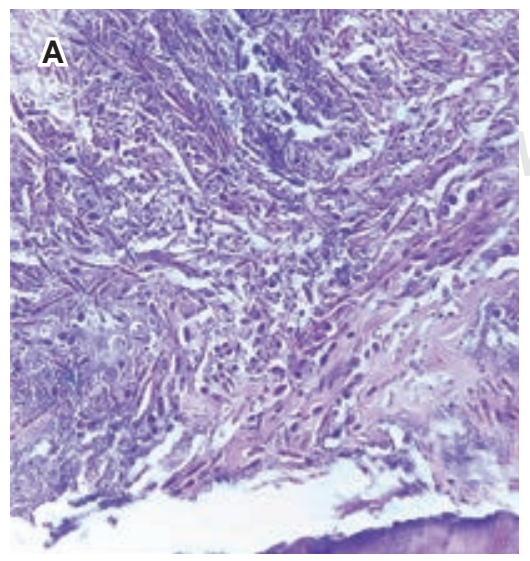

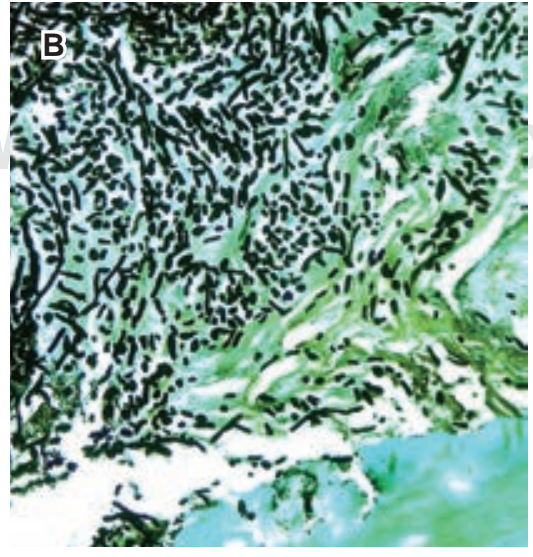

Figura 3:

A) Fotomicrografía H\&E a 200x en la que se observa proliferación de estructuras celulares cilíndricas, alargadas no septadas, algunas en ángulos de $90^{\circ}$, compatibles con hifas. B) Tinción Grocott positivo 200x. 
dejando un defecto de brown tipo III de manera bilateral. El procedimiento se efectuó bajo anestesia general con intubación orotraqueal con tubo armado no. 8, se inició asepsia y antisepsia, colocación de campos estériles, colocación de tapón faríngeo, marcaje de abordajes quirúrgicos en tejidos blandos tipo Weber Ferguson bilateral, se infiltró lidocaína con epinefrina 1:100,000. Se realizó tarsorrafia bilateral, abordaje tipo Weber Ferguson bilateral (Figura 4), disecando por planos hasta llegar a nivel óseo, se observó perforación de pared anterior de seno maxilar izquierdo y en base nasal bilateral, se continuó disecando hasta la mucosa palatina que se observaba clínicamente sana, se marcaron y realizaron las osteotomías tipo Le Fort I conservando rin orbitario bilateral y se perforó el piso de órbita del lado izquierdo, por lo que se colocó una malla de titanio fijada con dos tornillos del sistema $1.5 \times 5 \mathrm{~mm}$ a nivel de reborde orbitario. Se realiza hemostasia y se sutura por planos con Vicryl 3-0. Se ingresa a la unidad de cuidados intensivos para manejo y control de vía aérea por probable colapso de tejidos blandos en orofaringe y nasofaringe.

La pieza quirúrgica es enviada al departamento de patología, medicina bucal y maxilofacial para su estudio histopatológico (Figura 5), reportando «mucormicosis maxilar bilateral». Posteriormente ingresa a piso, a cargo del servicio de cirugía maxilofacial y medicina interna bajo el mismo esquema con anfotericina $b$ deoxicolato. Se hicieron lavados quirúrgicos y cambios de apósito palatino cada tres días.

Se tomó nueva biopsia de mucosa palatina por presentar una zona eritematosa; el reporte emitido fue «tejidos blandos con inflamación abscedada, colonización bacteriana y necrosis». Se realiza tinción de PAS resultando negativo para microorganismos fúngicos. Es revalorado por el servicio de infectología, quienes sugieren continuar con tratamiento y vigilancia. Se egresó paciente del hospital al cumplir el tratamiento de 26 días y no mostrar datos de persistencia de la infección.

Paciente actualmente sin datos de recidivas, a 16 meses de seguimiento, con presencia de fístula palatonasal izquierda sin datos de secreción purulenta ni necrosis, se encuentra en protocolo de colocación de implantes cigomáticos y rehabilitación protésica (Figura 6).

\section{DISCUSIÓN}

La mucormicosis es una infección micótica aguda oportunista y con consecuencias fatales, es causa- da por varios tipos de mohos filamentosos que se encuentran ampliamente distribuidos en todo el mundo. Actualmente se considera la tercera micosis invasiva más común luego de la candidiasis y la aspergilosis; se ha incrementado de manera significativa en las últimas dos décadas y puede afectar cualquier órgano del cuerpo. Predomina en el sexo masculino a una razón 6:4 con respecto al sexo femenino y tiene mayor incidencia en adultos jóvenes entre la segunda y cuarta décadas de la vida. ${ }^{5,6}$

Los hongos responsables de la mucormicosis pertenecen a la clase de los Mucoromycotina del orden de los mucorales representados por varios géneros, entre ellos los géneros Mucor, Rhizopus, Rhizomucor y Absidia. Al microscopio se destacan por sus hifas anchas (5-15 micras), aceptadas y con ramificación irregular. De todas las formas clínicas, la MROC es la más frecuente. Usualmente es causada por el hongo Rhizopus oryzae y se caracteriza por cuadros de sinusitis aguda con fiebre, cefalea, epifora y congestión nasal con una evolución generalmente rápida a través de la diseminación vascular a las estructuras contiguas hasta el parénquima cerebral. ${ }^{7-9}$

La diabetes mellitus se considera un factor de predisposición en $60-81 \%$ de los casos, aunque en pacientes trasplantados renales ha sido reportada como la primera causa. Los pacientes con diabetes mellitus tipo 1 y 2 tienen alteraciones celulares y humorales que los predispone a procesos infecciosos de diversa índole. Entre los más importantes destacan la disminución de la actividad fagocítica por parte de los neutrófilos y la incapacidad de opsonización, que son parte de la defensa celular principal contra los mucorales. Esta disfunción neutrofílica más el pH sérico en niveles bajos disminuyen el efecto fagocítico de los macrófagos y el efecto quimiotáctico y oxidativo de los neutrófilos. Resulta fundamental la modificación del sistema de transferrina que, en los casos de cetoacidosis diabética, debido a la acidez del medio, condiciona mayor concentración de hierro libre $\left(\mathrm{Fe}^{2+}\right)$ que es utilizado por los mucorales, reduciendo su actividad inhibitoria. Se ha demostrado que los cuerpos cetónicos alteran la permeabilidad de la barrera hematoencefálica, esto permite contribuir a la afección cerebral. Los mucorales aprovechan específicamente ambas condiciones, pues poseen un complejo enzimático denominado cetona-reductasa, que se activa en acidosis y metaboliza los cuerpos cetónicos. ${ }^{10-13}$ 


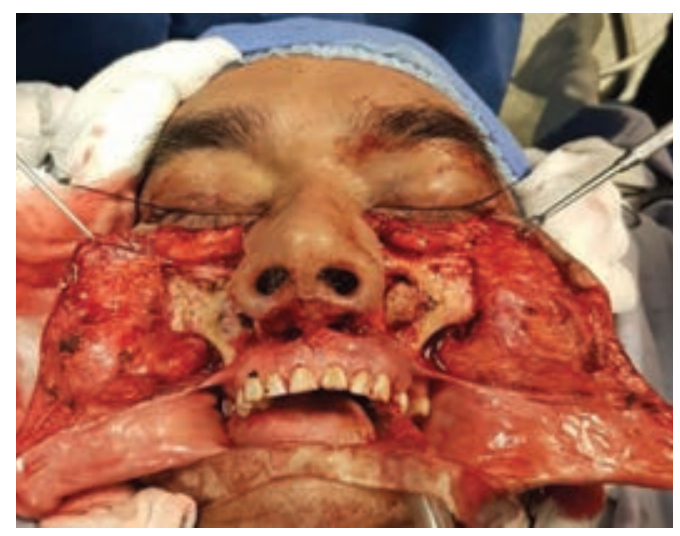

Figura 4: Abordaje Weber Ferguson bilateral, se diseca por planos hasta llegar a nivel subperióstico y exposición ósea. Se encuentra perforación de pared anterior de seno maxilar izquierdo.

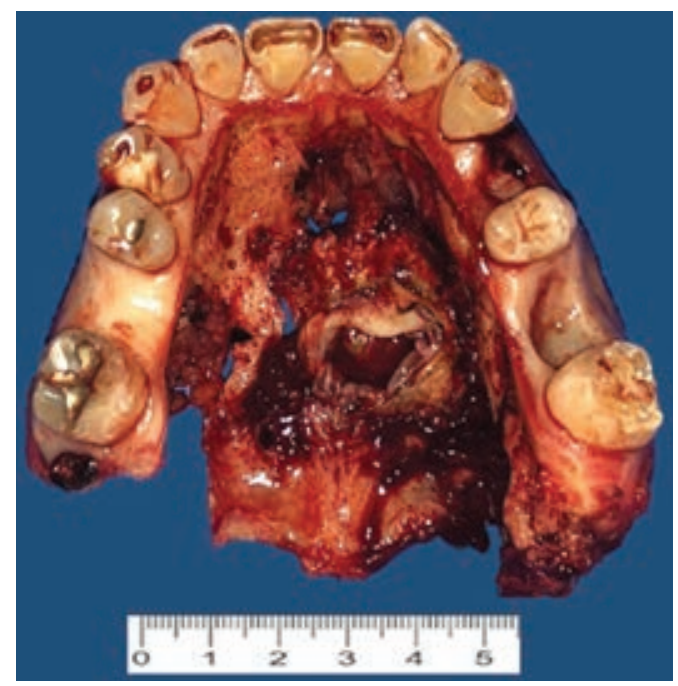

Figura 5: Pieza quirúrgica. Espécimen producto de maxilectomía total bilateral, corresponde a hueso maxilar con dimensiones totales de $7.0 \times 5.8 \times 5.3 \mathrm{~cm}$, se identifican órganos dentarios implantados en la apófisis alveolar en la mucosa palatina, se identifica úlcera de $1.8 \times 1.7 \times 1.5 \mathrm{~cm}$ y algunas perforaciones en la apófisis palatina del maxilar.

El diagnóstico de la mucormicosis siempre requiere confirmación histológica, los estudios de imagen pueden sugerir el compromiso anatómico por dicha infección, pero no son diagnósticos. Sin embargo, por la magnitud del compromiso, la rápida progresión, la invasión local y las implicaciones potencialmente fatales se requiere un alto índice de sospecha y en el contexto clínico adecuado estaría indicado el inicio temprano del manejo médico y quirúrgico aun sin la confirmación histológica. . $^{13,14}$
El tratamiento de la MROC se basa en cuatro principios fundamentales. En primer lugar, se debe hacer un diagnóstico temprano basado en un alto índice de sospecha. En segundo lugar, el inicio rápido del tratamiento, tanto médico como quirúrgico, idealmente en los primeros cinco días ha demostrado menor tasa de mortalidad a tres meses. En tercer lugar, es importante eliminar el factor predisponente, en particular el uso de glucocorticoides que debería ser minimizado o lo ideal sería suspenderlo. En cuarto lugar, el pilar más importante en el manejo de la mucormicosis rinocerebral es el desbridamiento quirúrgico amplio de los tejidos comprometidos, cuyo objetivo es cortar la cadena entre la angioinvasión, la necrosis y la mala penetración de los medicamentos al sitio de infección. En general el tratamiento antifúngico consiste en la administración de polienos como la anfotericina $B$ en sus formas deoxicolato $o$ liposomal en asociación con posaconazol. ${ }^{13}$

La duración del tratamiento médico debe ser individualizada esperando la resolución tanto clínica como radiográfica. Se debe considerar que a pesar del manejo adecuado y temprano, la mortalidad por esta infección micótica es de alrededor de $45 \%$ en la forma local, pero llega hasta una mortalidad de $93 \%$ en la forma rinocerebral. ${ }^{12-14}$

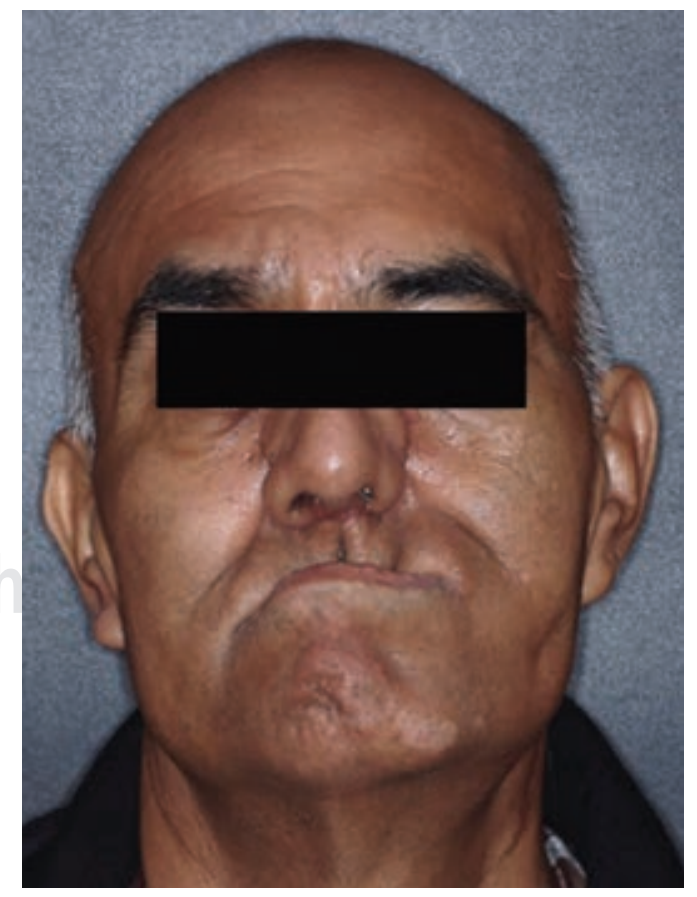

Figura 6: Fotografías postquirúrgicas de seguimiento, vista frontal. 


\section{CONCLUSIÓN}

La mucormicosis debe considerarse como diagnóstico diferencial en úlceras localizadas en paladar en todo paciente inmunosuprimido, especialmente en pacientes diabéticos no controlados con cetoacidosis a fin de evitar complicaciones y su progresión a una mucormicosis rino-órbito-cerebral que tiene una alta morbimortalidad con secuelas fatales. Es vital el diagnóstico y tratamiento oportuno para disminuir la morbimortalidad. El tratamiento quirúrgico debe ser radical con márgenes libres amplios para evitar la persistencia de la infección.

\section{BIBLIOGRAFÍA}

1. Cofré F, Villarroel M, Castellón L, Santolaya ME. Tratamiento exitoso de una mucormicosis rinocerebral persistente en un paciente pediátrico durante el debut de una leucemia aguda. Rev Chil Infectol. 2015; 32 (4): 458-463.

2. Artal R, Ágreda B, Serrano E, Alfonso JI, Vallés H. Mucormicosis rinocerebral: a propósito de ocho casos. Acta Otorrinolaringol Esp. 2010; 61 (4): 301-305.

3. Berdai MA, Labib S, Harandou M. Mucormycose rhinocérébrale compliquant une acidocétose diabétique. Presse Med. 2016; 45 (1): 145-146. doi: 10.1016/j.lpm.2015.11.002.

4. Di Carlo P, Pirrello R, Guadagnino G, Richiusa P, Lo Casto $A$, Sarno $C$ et al. Multimodal surgical and medical treatment for extensive rhinocerebral mucormycosis in an elderly diabetic patient: a case report and literature review. Case Rep Med. 2014; 2014: 527062.

5. Dimaka K, Mallis A, Naxakis SS, Marangos M, Papadas TA, Stathas $\mathrm{T}$ et al. Chronic rhinocerebral mucormycosis: a rare case report and review of the literature. Mycoses. 2014; 57 (11): 699702.

6. Kim JG, Park HJ, Park JH, Baek J, Kim HJ, Cha IH et al. Importance of immediate surgical intervention and antifungal treatment for rhinocerebral mucormycosis: a case report. J Korean Assoc Oral Maxillofac Surg. 2013; 39 (5): 246-250. doi: 10.5125/ jkaoms.2013.39.5.246.

7. Kolekar JS. Rhinocerebral mucormycosis: a retrospective study. Indian J Otolaryngol Head Neck Surg. 2015; 67 (1): 93-96.

8. Kursun E, Turunc T, Demiroglu YZ, Alışkan HE, Arslan AH. Evaluation of 28 cases of mucormycosis. Mycoses. 2015; 58 (2): 82-87.

9. Mattingly JK, Ramakrishnan VR. Rhinocerebral mucormycosis of the optic nerve. Otolaryngol Head Neck Surg. 2016; 155 (5): 888889.

10. Reddy SS, Rakesh N, Chauhan P, Sharma S. Rhinocerebral mucormycosis among diabetic patients: an emerging trend. Mycopathologia. 2015; 180 (5-6): 389-396.

11. Royer M, Puéchal X. Mucormycosis in systemic autoimmune diseases. Joint Bone Spine. 2014; 81 (4): 303-307. doi: 10.1016/j. jbspin.2014.01.002.

12. Singh V, Singh M, Joshi C, Sangwan J. Rhinocerebral mucormycosis in a patient with type 1 diabetes presenting as toothache: a case report from Himalayan region of India. BMJ Case Rep. 2013; 2013: bcr2013200811. doi: 10.1136/bcr-2013200811.

13. Sravani T, Uppin SG, Uppin MS, Sundaram C. Rhinocerebral mucormycosis: pathology revisited with emphasis on perineural spread. Neurol India. 2014; 62 (4): 383-386. doi: 10.4103/00283886.141252.

14. Vaezi A, Moazeni M, Rahimi MT, de Hoog S, Badali H. Mucormycosis in Iran: a systematic review. Mycoses. 2016; 59 (7): 402-415.

Conflicto de intereses: No hay conflicto de intereses. 